\title{
On the thickness uniformity of micropatterns of hyaluronic acid in a soft lithographic molding method
}

\author{
Hoon Eui Jeong and Kahp Y. Suh ${ }^{\text {a) }}$ \\ School of Mechanical and Aerospace Engineering, Seoul National University, Seoul 151-742, Korea
}

(Received 13 September 2004; accepted 12 April 2005; published online 2 June 2005)

\begin{abstract}
A soft lithographic molding is a simple and yet robust method for fabricating well-defined microstructures of a hydrophilic biopolymer such as polyethylene glycol and polysaccharide over a large area. The method consists of three steps: placing a polydimethylsiloxane mold with a bas-relief pattern onto a drop-dispensed polymer solution typically dissolved in water, letting the mold and the solution undisturbed in contact until solvent evaporates completely, and leaving behind a polymer replica after mold removal. In such a molding process, water can only evaporate from the edges of the mold due to impermeable nature of polydimethylsiloxane to water, resulting in a nonuniform distribution of film thickness or pattern height. Here we examine systematically how the evaporation rate affects the thickness distribution of the resulting microstructures by evaporating the solution of hyaluronic acid in various conditions. To compare with a theory, we also present a simple theoretical model based on one-dimensional conservation equation for a liquid film, which is in good agreement with the experimental data. (C) 2005 American Institute of Physics.
\end{abstract}

[DOI: 10.1063/1.1929095]

\section{INTRODUCTION}

Soft lithography is a convenient route to fabricating templates or microstructures for biological applications due to its ability to control the molecular structure of surfaces and to fabricate channel structures appropriate for microfluidics, and to pattern and manipulate cells. ${ }^{1}$ In particular, biological applications often involve relatively large feature sizes $(>50 \mu \mathrm{m})$, which is suitable for soft lithography.

Recently, micromolding of a hydrophilic biopolymer such as hyaluronic acid (HA) (Refs. 2 and 3) or polyethylene glycol $(\mathrm{PEG}),{ }^{4-8}$ which is a form of soft lithography, has been introduced as an alternative to microcontact printing $(\mu \mathrm{CP})$ for the development of protein and cell arrays. In previous work, we reported a direct molding method using polydimethylsiloxane (PDMS) mold, which involves the placement of a patterned PDMS mold on top of a dropdispensed or spin-coated HA/PEG solution typically dissolved in water or ethanol, leaving behind a polymer replica after solvent evaporation followed by mold removal. In comparison with $\mu \mathrm{CP}$, the technique generates features varying in height with precise control over cell migration and interconnection of cell arrays. ${ }^{6}$

If water is used as a solvent, the wetting environment is much different from previous studies of micromolding of hydrophobic polymers such as polystyrene involving organic solvents. ${ }^{9}$ For example, instead of filling into the mold, a water solution under the void space recedes downwards ("capillary depression") until the substrate surface becomes exposed ("dewetting") due to an obtuse contact angle of water on PDMS mold $\left(\sim 105^{\circ}\right)$ (Ref. 10) whereas hydrophobic polymers rise into the void space ("capillary rise") by par-

\footnotetext{
a) Author to whom correspondence should be addressed; electronic mail: sky4u@snu.ac.kr
}

tially wetting the PDMS wall (acute contact angle). This phenomenon can be described by well-known Young's equation. ${ }^{11}$

A potential concern with this micromolding is that the thickness profile after the molding process could be nonuniform. We observe experimentally higher microstructures at the edge of the mold, as water cannot be absorbed into the mold, evaporate through the mold, but can only evaporate from the edges of the mold due to impermeable nature of PDMS to water. It is also observed that the height of microstructure is maximal at the edge, while decreasing monotonically to the center as shown and discussed shortly.

In this paper, we examine systematically how the evaporation rate affects the thickness distribution of the microstructures of hyaluronic acid (HA). HA is chosen because it is one of the most important and frequently used biocompatible biopolymer in various applications such as drug delivery and tissue engineering. ${ }^{12,13}$ Furthermore, HA is well soluble in water and forms a viscous solution that can be treated easily without much caution. We also present a simple theoretical model based on one-dimensional conservation equation to describe the thickness distribution for various evaporation rates.

\section{EXPERIMENTAL METHOD}

For the experiment, we fabricated a PDMS mold by casting PDMS (Sylgard 184 elastomer, Dow Corning) against a silicon master prepared by photolithography (10\% curing agent). Then the prepolymer was well mixed and incubated at $70{ }^{\circ} \mathrm{C}$ for $1 \mathrm{~h}$. After curing, PDMS molds were detached from the master and cut into $1.5 \mathrm{~cm}^{2}$ prior to use. A few drops of the solution of HA (Genzyme Inc., $M_{n}=2.1 \mathrm{MDa}$ ) $(\sim 50 \mu \ell)$ at a concentration of $0.5 \mathrm{~g} / 100 \mathrm{ml}$ in distilled water were placed on a glass slide and a PDMS mold of $0.876 \mathrm{~g}$ having $70-\mu \mathrm{m}$ circles (features sticking in) was subsequently 


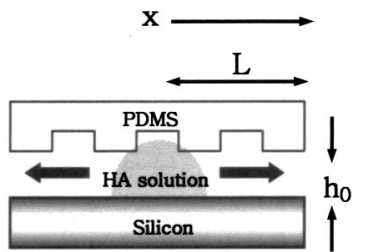

Spreading of solution

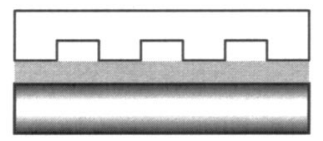

Evap. + Dewetting
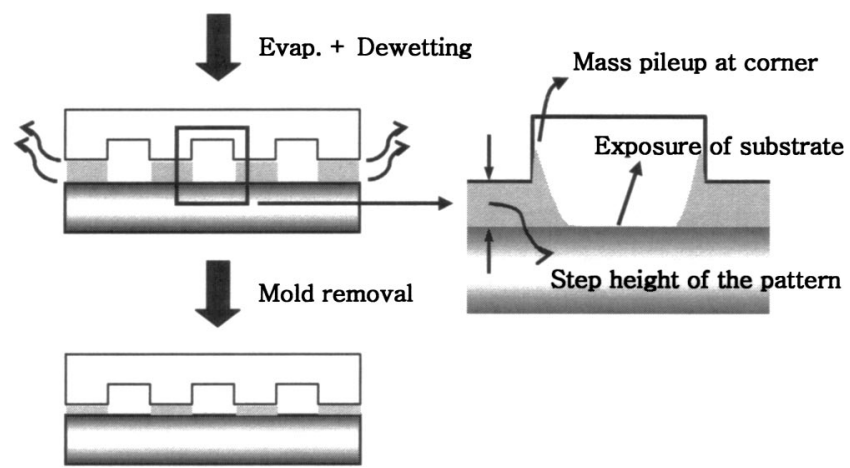

FIG. 1. A schematic of the experimental procedure where water evaporates only from the edge of the mold due to impermeable nature of PDMS mold to water. The pileup of HA during the dewetting process and the definition of the step height are shown in the box.

placed on top of the solution without any additional pressure, resulting in the spontaneous spreading of the solution. The drop-dispensing method was effective in reducing the number of bubbles that formed at the time of contact. The viscosity of the solution and the average thickness of HA after complete solvent evaporation were measured to be $\sim 4.5 \mathrm{~Pa} \mathrm{~s}$ (RMS800, Rheometrics Inc.) and $\sim 170 \mathrm{~nm}$, respectively. To control the evaporation rate, the samples were stored at various conditions (sealing in a petri dish at $27^{\circ} \mathrm{C}$, no sealing at $27^{\circ} \mathrm{C}$, and $40^{\circ} \mathrm{C}$ ). It is noted that temperature cannot be raised above $40{ }^{\circ} \mathrm{C}$ because of higher degree of HA crystallization. After $12 \mathrm{~h}$, the mold was peeled off and atomic force microscopy (AFM, Dimension 3100, Digital Instru- ment) images of the HA microstructures were taken in the tapping mode and the images were flattened and further processed if necessary. The images of PDMS mold were taken by scanning electron microscopy (SEM, XL30FEG, Philips Electron Co.) measurements. A schematic diagram of the experimental procedure is shown in Fig. 1.

\section{RESULTS AND DISCUSSION}

As shown in Fig. 1, dewetting takes place at the bottom of the substrate as evaporation proceeds, resulting in a complete exposure of the substrate surface. This exposure of the substrate is essential for subsequent selective deposition of proteins or cells as reported previously. ${ }^{2,3}$ Based on a surface energy consideration, any capillary rise is very unlikely since the contact angle of water on PDMS is $105^{\circ}$. In the course of dewetting, the solution dewets from the substrate and appears to move to the periphery of the circle's corners to minimize the surface-to-volume ratio. Protruding features are present at the corner as a result of dewetting and mass pileup. The step height of the pattern is then defined as the distance between the flat region of HA and the substrate, as shown in Fig. 1.

Shown in Fig. 2 is a typical optical micrograph of the molded HA microstructure along with a SEM image of PDMS mold used in the experiment. The height of the void space was about $70 \mu \mathrm{m}$ such that it is nearly impossible for the solution to touch the ceiling of the void space. The nonuniform distribution of film thickness is well represented by the difference in contrast with an optical micrograph in Fig. 2(a). It is noted in this regard that the microstructure is relatively flat when other organic solvents such as ethanol or methanol are used since these organic solvents are readily absorbed into the PDMS mold and evaporates into air by diffusion through the mold. ${ }^{14}$ For biological applications, this nonuniformity is potentially detrimental to the construction of large-area arrays of biological species and control of cell migration or interconnection. Figure 2(c) illustrates the thickness profile that results after complete evaporation of (a)

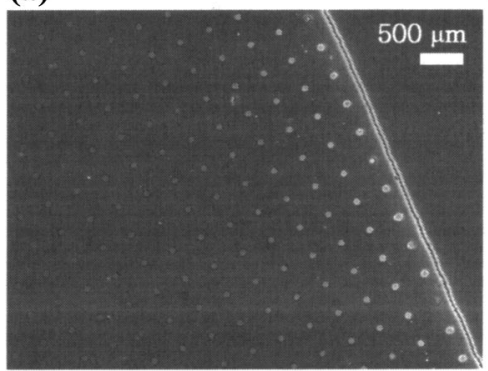

(b)

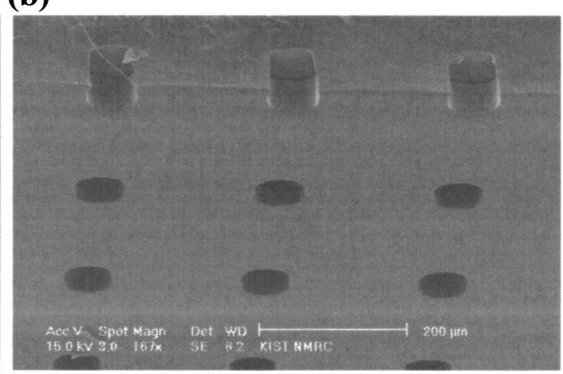

FIG. 2. (a) A typical optical micrograph of the molded HA microstructure (70- $\mu$ m circles). (b) A SEM image of the PDMS mold used in the experiment. (c) An illustration depicting the thickness distribution after solvent evaporation.

(c)

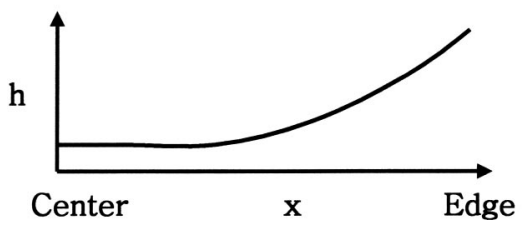




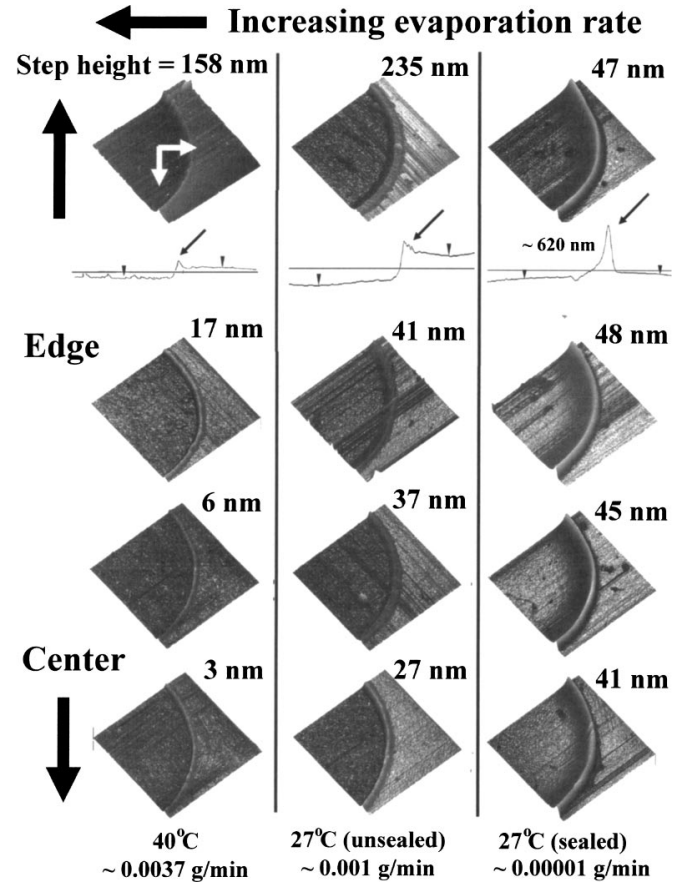

FIG. 3. Three-dimensional AFM images of the HA microstructures with three evaporation conditions at four different locations from center to edge (equal spacing). The numbers in the figure indicate the step height of the pattern. Cross-sectional AFM images are shown for the first low, which clearly indicate the presence of the pileup of HA around the circle. The arrows indicate the pileup of HA at the edge of the circle.

the solvent. As discussed shortly, this parabolic profile occurs at relatively fast evaporation rates including at ambient conditions.

Figure 3 shows AFM images of the resulting microstructures for the three evaporation conditions. The numbers in the figure indicate the step height of the pattern and the four images were obtained at equal distance from center to edge for each evaporation condition. The average evaporation rates were measured in a separate experiment by measuring the amount of evaporated water as a function of time, yielding $\sim 10^{-5}, \sim 10^{-3}$, and $\sim 3.7 \times 10^{-3} \mathrm{~g} / \mathrm{min}$, respectively (increasing order). A number of findings are drawn from Fig. 3. First, in terms of the step height the thickness profile becomes more nonuniform as the evaporation rate increases whereas it becomes more uniform as the evaporation rate decreases. A qualitative explanation for this is that if the evaporation rate is relatively fast, the overall process becomes diffusion limited, i.e., water evaporates instantaneously right after it reaches the edge of the mold. This diffusion-limited process would result in a nonuniform distribution, as shown in the figure. On the other hand, when water evaporates at $27{ }^{\circ} \mathrm{C}$ while sealed in a petri dish, a very uniform thickness profile was observed irrespective of the position, as shown in the third column of Fig. 3. In this case, the rate of evaporation would be relatively slower than that of diffusion and thus the overall process becomes evaporation limited. Interestingly, the average height of the pattern was dramatically reduced in comparison with other microstructures at the edge, except for protruding features around the circle. The height of the protruding features increases with a decreasing in the evaporation rate, rendering a height as high as $\sim 620 \mathrm{~nm}$ for the slowest evaporation in the third column. We hypothesize that the pileup of HA at the corner is thermodynamically stable to minimize the surface-tovolume ratio and the slow evaporation might provide enough time to reach a thermodynamic equilibrium. At moderate or fast evaporation, there would be a competition between mass pileup along the PDMS wall and lateral diffusion towards the edge of the mold so that the protrusion would not be so significant. The protruding features are potentially useful to control cell migration or interconnection for cell arrays since they could be used as diffusion barriers. Although not shown, we observed a similar behavior when a PEG polymer was used instead of HA.

To gain an understanding on the spatial distribution of polymer during the micromolding process, we consider a one-dimensional conservation equation for a liquid film: ${ }^{15}$

$$
\eta \frac{\partial h}{\partial t}=-\frac{\gamma}{3} \frac{\partial}{\partial x}\left(h^{3} \frac{\partial^{3} h}{\partial x^{3}}\right),
$$

where $\eta$ is the viscosity, $\gamma$ is the surface tension, and $h$ is the height or thickness of the HA layer. The conversation equation described the motion of a liquid film in response to a change in the boundary condition at the edge, i.e., the evaporation imposes a boundary condition of the constant gradient of film thickness as shown shortly. Since water is continuously depleted from the solution, it could be convenient for mathematical manipulation to apply mass conservation to solute only (HA in this case). Therefore, we define $h$ as the height of HA layer without water, which can be justified as long as HA concentration is maintained to be the same across the axial direction during the evaporation process. Our further assumptions are as follows: first, the diffusion of HA molecules inside the liquid solution is neglected in the current model because the motion of water is of interest. Second, the interactions between HA molecules and the PDMS mold are negligible. Third, the initial thickness profile of the HA solution is completely flat at time zero and there is no evaporation or dewetting before the spreading of the solution is finished. Fourth, any capillarity along the PDMS wall does not affect the final thickness profile. Fifth, the diffusion and evaporation rate constants of water in HA solution are not affected by the presence of HA molecules. These assumptions may be valid for a very dilute solution of HA used in this study $(\sim 0.5$ wt $\%)$.

Equation (1) is highly nonlinear and thus solving the equation requires significant computational effort using numerical methods. For mathematical simplicity, we employ an even polynomial of fourth order considering the symmetric thickness profile at the origin, which is given by

$$
h(x, t)=h_{c}(t)+a(t) x^{2}+b(t) x^{4},
$$

where $h_{c}(t)$ is the thickness at the center, which is taken as the origin of the $x$ axis (see Fig. 1), and $a(t)$ and $b(t)$ are the time-dependent coefficients of the polynomial. The coefficients are determined by two boundary conditions:

$$
\left.\frac{d h}{d x}\right|_{x=L}=K_{e} \cdot h_{L},
$$



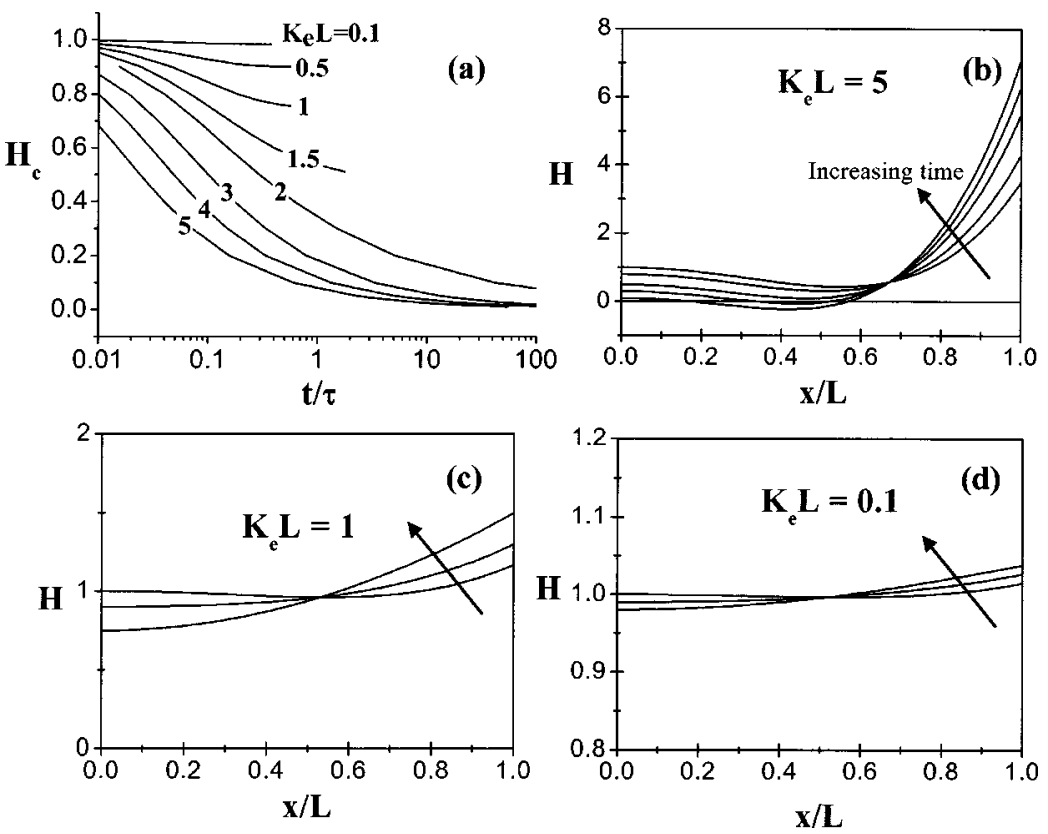

FIG. 4. (a) The change of film thickness at the center $\left(H_{c}\right)$ as a function of time $(t / \tau)$ for various $K_{e} L$ values. (b)-(d) The corresponding thickness profiles $(H)$ along the axial direction $(x / L)$. Lower $K_{e} L$ values provide more uniform microstructures.

$$
\int_{0}^{L} h(x, t) d x=h_{0} L .
$$

The first condition indicates that the diffusion flux at the edge ( $x=L$ where $L$ is the half the mold size) is equal to the evaporation rate that is proportional to the amount of solution. The parameter, $K_{e}$, is defined as $k_{e} / D$ where $k_{e}$ is the evaporation rate constant of water for the squeezing process presented in this study and $D$ is the diffusion coefficient of water. The second condition indicates mass balance of HA throughout the molding process where $h_{0}$ is the initial film thickness of the HA layer (not the thickness of the solution). Inserting the two boundary conditions into Eq. (1) with an algebraic manipulation yields

$$
\begin{aligned}
& a(t)=\frac{15\left(K_{e} L-4\right)}{2\left(K_{e} L-7\right) L^{2}} h_{0}-\left(6+\frac{12}{K_{e} L-7}\right) \frac{h_{0}(t)}{L^{2}}, \\
& b(t)=\frac{15\left(2-K_{e} L\right)}{2\left(K_{e} L-7\right) L^{4}} h_{0}+\frac{5\left(K_{e} L-3\right)}{\left(K_{e} L-7\right)} \frac{h_{0}(t)}{L^{4}} .
\end{aligned}
$$

Thus, we have

$$
\begin{aligned}
\frac{h(x, t)}{h_{0}}= & \frac{h_{c}(t)}{h_{0}}+\left[\frac{15\left(K_{e} L-4\right)}{2\left(K_{e} L-7\right)}-\left(6+\frac{12}{K_{e} L-7}\right) \frac{h_{c}(t)}{h_{0}}\right] \\
& \times\left(\frac{x}{L}\right)^{2}+\left[\frac{15\left(2-K_{e} L\right)}{2\left(K_{e} L-7\right)}+\frac{5\left(K_{e} L-3\right)}{K_{e} L-7} \frac{h_{c}(t)}{h_{0}}\right] \\
& \times\left(\frac{x}{L}\right)^{4} .
\end{aligned}
$$

Equation (6) indicates that the overall thickness profile is determined from three dimensionless parameters, $h_{c}(t) / h_{0}$, $K_{e} L$, and $x / L$, so that a dimensionless version of the equation is easily obtained.

In parallel, $h_{c}(t)$ can be evaluated in Eq. (1) at $x=0$, which gives

$$
\begin{aligned}
\frac{\partial h_{c}}{\partial t} & =-\frac{8 \gamma b(t)}{\eta} h_{c}(t)^{3} \\
& =-\frac{8 \gamma h_{c}(t)^{3}}{\eta L^{4}}\left[\frac{15\left(2-K_{e} L\right)}{2\left(K_{e} L-7\right)}+\frac{5\left(K_{e} L-3\right)}{K_{e} L-7} \frac{h_{c}(t)}{h_{0}}\right] .
\end{aligned}
$$

Integrating Eq. (7) by part yields

$$
\int_{H_{c}}^{1} \frac{1}{H_{c}^{3}\left\{\frac{15\left(2-K_{e} L\right)}{2\left(K_{e} L-7\right)}+\frac{5\left(K_{e} L-3\right)}{\left(K_{e} L-7\right)} H_{c}\right\}} d H_{c}=8 \frac{t}{\tau},
$$

where $H_{c} \equiv h_{c}(t) / h_{0}$ and $\tau$ is the characteristic time that is given by $\eta L^{4} / \gamma h_{0}^{3}$. According to the definition of the characteristic time, it takes much time for the film to flow as the viscosity increases and the film thickness decreases, which is readily understood in terms of mobility of the film.

Figure 4(a) shows the decrease of film thickness at the center $\left(H_{c}\right)$ with time progression $(t / \tau)$ for various $K_{e} L$ values along with the corresponding thickness profiles [Figs. 4(b)-4(d)]. The value of $h_{0}$ can be obtained graphically from the resulting thickness profile since the amount of HA is conserved. As expected, $H_{c}$ decreases with time, the rate of which strongly depends on the value of $K_{e} L$. Higher values $\left(K_{e} L>3\right)$ could give rise to nearly complete depletion of HA at the center [Fig. 3(a)], which agrees with few nanometer heights left behind on the surface in the first column of Fig. 3. Furthermore, lower $K_{e} L$ values (lower evaporation rate, higher diffusion rate, and smaller sample size) lead to relatively uniform step heights whereas higher $K_{e} L$ values (higher evaporation rate, lower diffusion rate, and larger sample size) cause the step height to be nonuniform.

A few comments are in order here regarding the definition of evaporation rate constant $k_{e}$. Since we are dealing with a confined film (sandwiched between the PDMS mold and the substrate), the evaporation occurs only at the edge in two dimensions. The bulk evaporation constant is typically defined as the evaporated amount of water per unit volume 


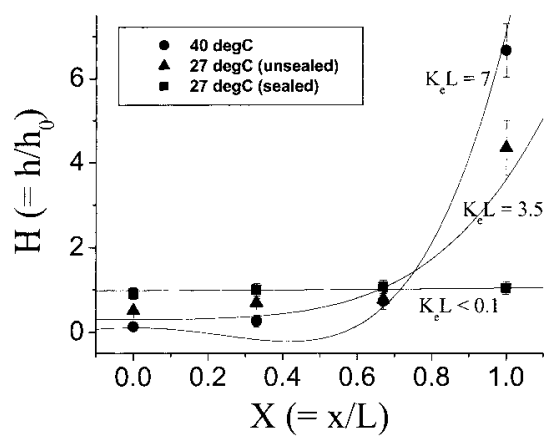

FIG. 5. Plotting the experimental data with theoretical predictions using $K_{e} L$ as an adjustable parameter. The change of curvature results from the use of a fourth-order polynomial, not reflecting the real situations.

as compared to unit area in the current system. Thus a relation between the two-dimensional and three-dimensional evaporation constants is given by

$$
k_{e}=k_{b} \times L,
$$

where $k_{b}$ is the evaporation rate constant for the bulk film, which was reported to be about $5 \times 10^{-5} \mathrm{~s}^{-1}$ at $27^{\circ} \mathrm{C}$. ${ }^{16}$ Since the sample size is typically on the order of $10^{-2} \mathrm{~m}$, the evaporation rate constant of the confined film would be two orders of magnitude smaller than that of the bulk film. Furthermore, since the autodiffusion coefficient of water is on the order of $10^{-8} \mathrm{~m}^{2} / \mathrm{s},{ }^{17}$ the value of $K_{e} L$ is on the order of 1 , which agrees with the experiment. It is noted in this regard that the evaporation rate and diffusion constants of water would be slightly different in the presence of HA molecules, which need to be clarified in a future study.

One can easily adjust the value of $K_{e} L$ to fit the experimental data in Fig. 3, which is shown in Fig. 5. The change of curvature in the case of higher $K_{e} L$ values $(>5)$ results from the use of a fourth-order polynomial in Eq. (2) and may not reflect the real situations. As discussed above, the value of $K_{e} L$ is estimated to be on the order of 1 at ambient conditions, which is in good agreement with 3.5 obtained at $27^{\circ} \mathrm{C}$. The evaporation rate constant for the sealed environment was lower than that for the unsealed environment by two orders of magnitude, as shown in Fig. 3. Accordingly, the corresponding value of $K_{e} L$ would be smaller by two orders of magnitude. It was found that the thickness distribution becomes flattened for $K_{e} L<\sim 0.1$ such that a direct comparison between theory and experiment is difficult for very slow evaporation. Nonetheless, the value of $K_{e} L$ for slow evaporation qualitatively agrees with the experiment.

\section{SUMMARY}

We have demonstrated that the uniform step height in the micromolding of a hydrophilic biopolymer can be achieved by utilizing slow evaporation of water in a sealed environment. The slow evaporation allows for an evaporationlimited process, thus providing enough time for the solution to diffuse and construct a flat, uniform height profile. Protruding structures were always present around the edge of the circle as a result of the pileup of HA during the dewetting process, which could be potentially useful to control cell migration and interconnections of cellular arrays. We also presented a simple one-dimensional conservation equation for a liquid film to describe the thickness profile for various evaporation rates. It turned out that the model is in good agreement with the experimental data with reasonable adjusting parameters. The ability to control surface topography is potentially useful to fabricate large-area arrays of proteins or cells on a surface or inside microfluidics channels.

This work was supported by the Ministry of Science and Technology through the Nanoscopia Center of Excellence at Seoul National University. This work was also partially supported by the Micro Thermal System Research Center of Seoul National University.

${ }^{1}$ G. M. Whitesides, E. Ostuni, S. Takayama, X. Y. Jiang, and D. E. Ingber, Annu. Rev. Biomed. Eng. 3, 335 (2001).

${ }^{2}$ K. Y. Suh, A. Khademhosseini, J. M. Yang, G. Eng, and R. Langer, Adv. Mater. (Weinheim, Ger.) 16, 584 (2004).

${ }^{3}$ A. Khademhosseini, K. Y. Suh, J. M. Yang, G. Eng, J. Yeh, S. Levenberg, and R. Langer, Biomaterials 25, 3583 (2004).

${ }^{4}$ G. Tae, R. G. H. Lammertink, J. A. Kornfield, and J. A. Hubbell, Adv. Mater. (Weinheim, Ger.) 15, 66 (2003).

${ }^{5}$ W. G. Koh, L. J. Itle, and M. V. Pishko, Anal. Chem. 75, 5783 (2003).

${ }^{6}$ A. Khademhosseini, S. Jon, K. Y. Suh, T. N. T. Tran, G. Eng, J. Yeh, J. Seong, and R. Langer, Adv. Mater. (Weinheim, Ger.) 15, 1995 (2003).

${ }^{7}$ K. Y. Suh and R. Langer, Appl. Phys. Lett. 83, 1668 (2003).

${ }^{8}$ K. Y. Suh, J. Seong, A. Khademhosseini, P. E. Laibinis, and R. Langer, Biomaterials 25, 557 (2004).

${ }^{9}$ Y. S. Kim, K. Y. Suh, and H. H. Lee, Appl. Phys. Lett. 79, 2285 (2001).

${ }^{10}$ E. Kim, Y. N. Xia, and G. M. Whitesides, Nature (London) 376, 581 (1995).

${ }^{11}$ A. W. Adamson and A. P. Gast, Physical Chemistry of Surfaces, 6th ed. (Wiley, New York, 1997).

${ }^{12}$ G. Abantangelo and P. Weigel, New Frontiers in Medical Science: Redefining Hyaluronan (Elsevier, Amsterdam, 2000).

${ }^{13} \mathrm{E}$. A. Balazs and J. L. Denlinger, in Clinical Uses of Hyaluronan: The Biology of Hyaluronan, edited by D. Evered and J. Welan (Wiley, New York, 1989), p. 265.

${ }^{14}$ J. N. Lee, C. Park, and G. M. Whitesides, Anal. Chem. 75, 6544 (2003).

${ }^{15}$ A. Oron, S. H. Davis, and S. G. Bankoff, Rev. Mod. Phys. 69, 931 (1997).

${ }^{16}$ J. H. Clint, P. D. I. Fletcher, and I. T. Todorov, Phys. Chem. Chem. Phys. 1, 5005 (1999).

${ }^{17}$ N. Rashidnia, R. Balasubramaniam, J. Kuang, P. Petitjeans, and T. Maxworthy, Int. J. Thermophys. 22, 547 (2001). 